\title{
RAMPing up voltage indicator imaging
}

\author{
Combining a voltage sensor with random-access microscopy allows imaging of neuronal activity in behaving mice.
}

G enetically encoded calcium indicators are widely used to read out the activity of neurons, but they neither report electrical activity directly, nor report sub-threshold activity that does not result in action potentials. This is where genetically encoded voltage indicators (GEVIs) can shine. However, imaging GEVIs is not trivial as they are expressed at lower levels than calcium indicators, because GEVIs are limited to the membrane. In addition, voltage events happen at faster time scales than calcium events, making it necessary to image at higher speed. These factors limit the photons available for imaging and require engineering advances on both the indicator and microscopy front.

Michael Lin from Stanford University, Stéphane Dieudonné from the École Normale Supérieure in Paris and their colleagues addressed this problem in a transatlantic collaboration. Lin and his team engineered the ASAP3 voltage sensor while Dieudonné and his team developed an ultrafast local volume excitation (ULoVE) approach, a variant of random-access multiphoton microscopy (RAMP). "The project is the result of an incredible amount of teamwork," says Lin.

ASAP 3 consists of a circularly permuted GFP variant and a voltage-sensing domain. To engineer ASAP3, Lin and his team started with an ASAP2 variant and conducted site-directed mutagenesis at multiple sites. To efficiently screen the mutants, Lin and his team set up an automated stimulation and image analysis system so that they could assess thousands of mutants a day. Lin credits the success of this part of the project to Ivan Dimov and Lagnajeet Pradhan. "Their creativity and apparent ability to survive on chips and pizza were crucial to this project," says Lin.

In addition to the hardware optimization, Mariya Chavarha in the Lin lab developed a new method for constructing and expressing GEVI variants, and figured out structurefunction relationships in ASAP2 to guide mutagenesis. "Some people might think tool development is mostly a matter of cranking through standard procedures in a low-tech manner," says Lin. But he assures that "a lot of new ideas and careful insight" were required for developing the ASAP family, including the earlier creation of ASAP1

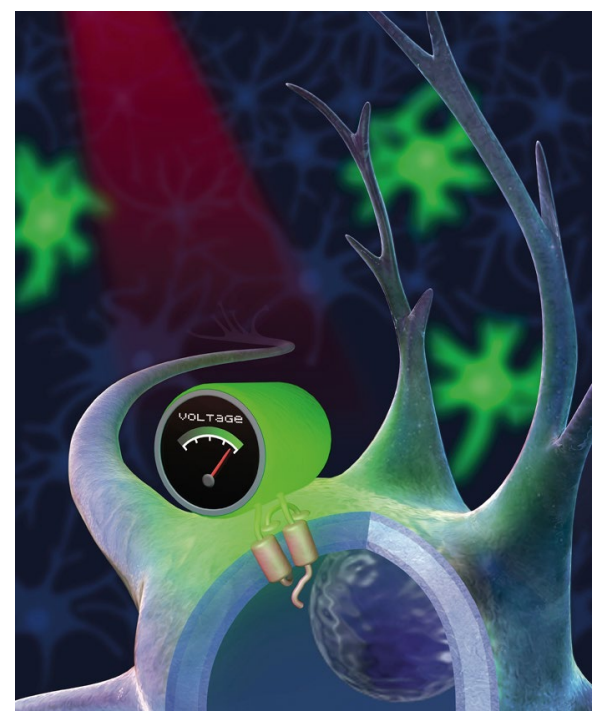

The ASAP3 voltage indicator reports voltage when excited by infrared light in two-photon microscopes. Credit: Layla Lang and Michael Z. Lin, Stanford University

and ASAP2s by former postdoc François St-Pierre.

A major advantage over many other GEVIs is that ASAP3 can be imaged under two-photon illumination conditions. ASAP3 responds to action potentials faster and with a larger response than ASAP2. "There are GEVIs that are brighter per molecule or have larger responses to voltage change or are faster, but ASAP3 is very good in all three at the same time, whereas other GEVIs lack one or two of the three essential characteristics. If this were gymnastics, you could say ASAP3 would win the all-around gold medal even while getting silvers in the individual events," says Lin.

Having a suitable GEVI in hand is not sufficient for in vivo imaging of voltage changes. The microscope technology needs to be optimized for high-speed imaging. For this, Dieudonnés expertise in randomaccess imaging came into play. RAMP is fast enough for imaging action potentials or subthreshold events, but with the inherently short dwell times the amount of photons that can be imaged in a diffraction-limited volume becomes limiting. Dieudonné and his team overcame this problem with ULoVE. "Instead of illumination in one diffraction-limited spot, you split your excitation into a small array of spots, which are then wiggled very quickly to scan through a volume within microseconds," explains Lin. Collecting photons from a larger volume also overcomes another problem of RAMP. Expanding the illuminated volume "makes the imaging less sensitive to micron-level brain motions that occur in vivo," says Lin.

Lin, Dieudonné and their colleagues applied ASAP3 and ULoVE in a variety of scenarios. They visualized electrical oscillations in mouse hippocampal neurons while the mouse was running on a treadmill. Such oscillations are thought to reflect the brain state. On top of these oscillations, the researchers could record action potentials. Furthermore, the researchers assessed how running influenced the membrane potential and firing rate in neurons in the primary visual cortex. They observed in several putative interneurons that firing rate increased during running, yet the baseline membrane potential was unchanged. In contrast, bursty neurons were either depolarized or hyperpolarized during locomotion.

In summary, "ASAP3 is useful for voltage imaging when you want to see spikes with precise timing or you need to detect spikes and subthreshold potential changes, using any imaging method. ULoVE, in particular, is useful when you want high [signal-to-noise ratio] for spikes and want to image for a long time or deeply in the brain," says Lin. Both he and Dieudonné will continue to improve the indicator and imaging technologies to increase sensitivity and throughput, respectively. But with the current state of the art, Lin thinks that "with our work and that of several other labs, the field of neuroscience is now finally able to observe the electrical events underlying intraneuronal and circuit computation in living brains, which has been a long-standing goal."

\section{Nina Vogt}

Published online: 6 January 2020 https://doi.org/10.1038/s41592-019-0720-4

Research paper

Villette, V. et al. Ultrafast two-photon imaging of a high-gain voltage indicator in awake behaving mice. Cell 179, 1590-1608.e23 (2019). 